\title{
The Role of Big Data Analytics in Developing System Dynamic Models
} Hamed Kianmehr, Ph.D. Candidate, Systems Science and Industrial Engineering, Binghamton
University, NY, USA http://orcid.org/0000-0002-7024-7864

Nasim Sabounchi, Assistant Professor, Systems Science and Industrial Engineering, Binghamton University, NY, USA http://orcid.org/0000-0002-8027-8779, email: sabounchi@binghamton.edu

Lina Begdache, Assistant Professor, Health and Wellness Studies, Binghamton University, NY, USA http://orcid.org/0000-0001-5213-6979

\begin{abstract}
Dietary factors are one of the risk factors that can impact the brain chemistry, which leads to mental distress. Based on our data mining approach, we found that mental distress in men is associated with eating unhealthy food. Our aim in this paper is to apply results from our big data analytics approach to inform system dynamics (SD) modeling to investigate the causal relationships between brain structures, nutrients from food and dietary supplements, and mental health. We perform descriptive analysis based on a large data set to estimate the SD modeling parameters. Finally, we calibrate the model towards a time series data collected for individuals on their dietary and distress patterns. The results reveal that bridging these different methodologies leads to further insights from the SD model and decreases the error of calibrated parameter values. Future research is needed to validate our initial results for investigating the relationship between mental distress and dietary intake.
\end{abstract}

Keywords: System Dynamics Modeling, Big data, Mental Distress, Diet

\section{Introduction}

Depression and anxiety are a significant mental health problem in the recent years. Studies show that human brain has links to various behavioral traits and different types of mood disorders [1]. A major factor that can affect the brain chemistry and mental health is nutrition [2]. Past studies that investigate the relationship between diet and mental well being have focused mainly on single nutrients [3]; however recent trends assess dietary patterns (DP). Furthermore, evidence shows that difference between men and women brain's functionally leads to different susceptibility for mental wellbeing [4, 5]. Many researches mentioned the gender morphological differences regarding brain structure which is explaining that men have a larger whole brain volume than women [6]. Furthermore, there is not agreement on the level of the grey and white matter between gender [7]. Grey matter (GM) is mainly in information-processing regions such as the hippocampus $(\mathrm{HC})$ and the amygdala of the limbic system. A research about analyzing differences in $\mathrm{HC}$, mentioned that men in all age 
groups has a larger $\mathrm{HC}$ volume in comparison of woman [8]. Additionally, the amygdala activation is not equal in man and women [9]. So, based on this discrepancy between brain structure of men and women and different effects of dietary patterns, it is very complex to study its relationship with mental distress. There are different methods to investigate the dietary patterns such as principal component analysis (PCA) and clustering, but limited research exists for deriving the dynamic relationship between DP and mental distress. Also here are several machine learning methods used in health care domain to investigate the latent factors [10].

The purpose of this study is to investigate the causal relationship between dietary variables and mental distress based on gender by adopting results from the big data analytics approach. To our knowledge, this is the first study, which explores how big data analytics can inform causal links in SD models. We build upon prior studies that investigate the association between mental distress based on dietary intake and differences among adult men and women.

\section{Method}

We apply machine learning techniques including PCA to derive the relationship between dietary pattern and mental distress. Then we use evidence from the literature to validate our findings. Based on our validated findings, we developed a system dynamics model to replicate time series data for one adult man between 30 to 39 years old that has recorded his dietary habits and mental distress levels over 40 days period. In order to estimate the unknown parameters, we applied traditional calibration methods in SD, that tries to decrease the difference between historical times series data and simulated results. Also, due to lack of data, we used an Indirect Inference approach by applying stepwise regression to estimate the other unknown parameters of the SD model [11].

\section{SD model}

We used evidence from the literature to validate our initial findings from PCA analysis. Then we develop system dynamics modeling based on these findings to depict the relationship between dietary intake and mental distress in men by using indirect inferences method. As shown in figure 1, Reinforcing loop1 (R1) captures the idea that the unhealthy diet (fast foods) has lack of essential nutrients (omega-3 fats and antioxidants), which is important for brain functionality and mental wellbeing. It means that when an adult man frequently consumes fast foods, over time, their brain structure changes and leads to higher anxiety and depression. A research conducted based on 5731 mid-old age participants reveals that unhealthy diet has a relationship with anxiety. Also due to aging and improper diet, HC volume decreases [12]. Loop R2 demonstrates that $\mathrm{HC}$ can affect neural circuits with regarding the emotion-related brain regions such as Amygdala. Then cortical-limbic circuit (CLC) can regulate the mood [13]. 
Loop R3 explains the effect of exercise on mental distress [14].

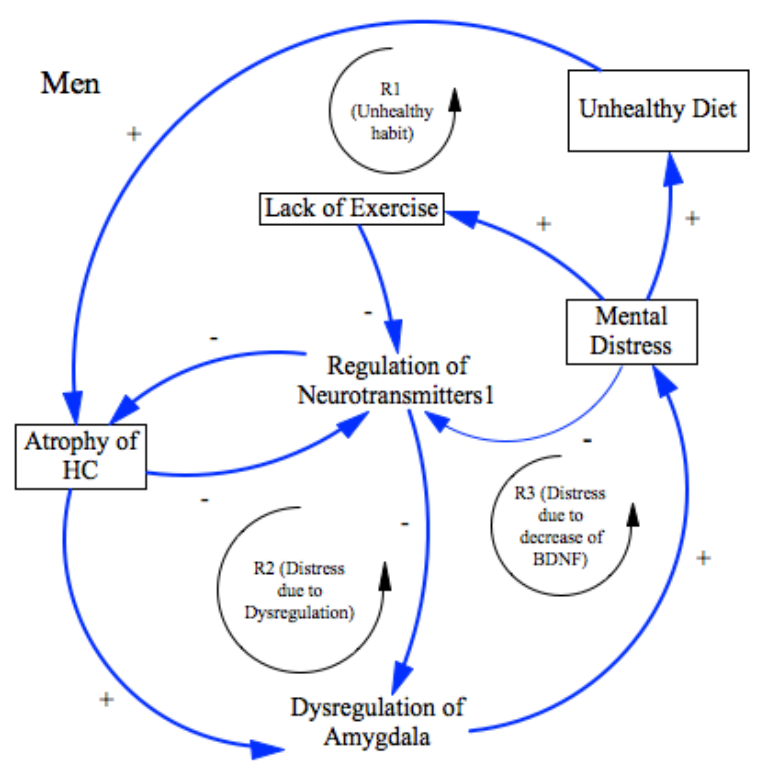

Figure 1 Casual Loop Diagram for men

\section{Results}

We represent a simple SD model of the main factors between diet and mental distress. As shown in figure 2, for men, there is a reinforcing loop which is defined by effect of fast food on mental distress and vice versa based on our article which is now under review. We applied the PCA method to derive the different dietary patterns based on 563 individuals for men versus women. Our key finding is that mental distress among men is dependent on fast food consumption and so we use traditional calibration tool to estimate $\theta_{1}$ and $\theta_{2}$ to find the relationship between fast food consumption and mental distress. Some other variables besides mental distress can impact individuals to eat more fast food which we consider them as exogenous variables and include breakfast, meat, exercise, and multivitamin. For estimating the unknown parameters that include $\theta_{3}, \theta_{4}, \theta_{5}, \theta_{6}$ and $\theta_{7}$, we run the stepwise regression to define the unknown parameters. 


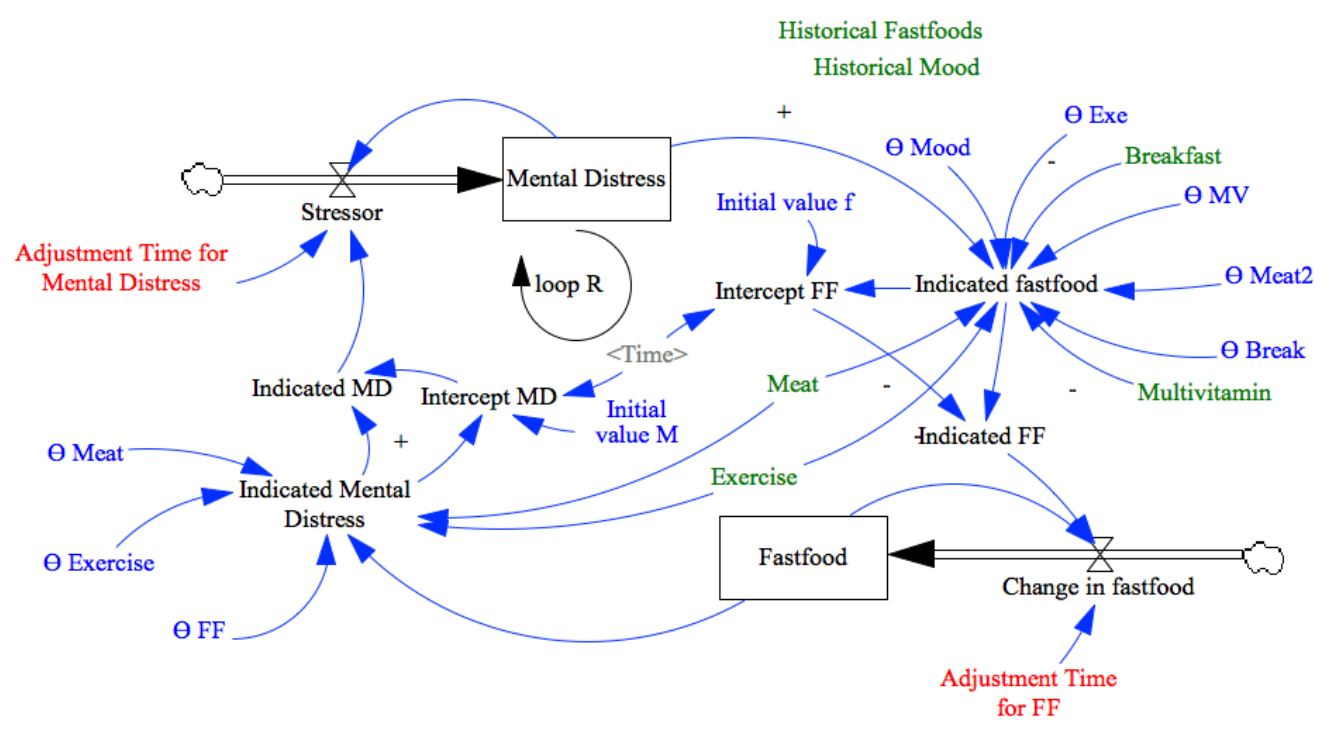

Figure 2 - SD model for unhealthy dietary intake and mental distress for Men 30 to 39 years

Table 1 Unknown Parameters in the SD Model

\begin{tabular}{|l|c|c|}
\hline Unknown parameters $(\theta)$ & Regression values & Calibrations values \\
\hline $\begin{array}{l}\text { Adjustment Time for Mental } \\
\text { Distress }\end{array}$ & & 1.859 \\
\hline Adjustment Time for FF & & 0.3 \\
\hline$\Theta$ Fast Food & -3.516 & \\
\hline$\Theta$ Mood & -0.01608 & \\
\hline$\Theta$ Exercise & -2.282 & \\
\hline$\Theta$ Multivitamin & 0.1228 & \\
\hline$\Theta$ Meat & 2.674 & \\
\hline$\Theta$ Meat2 & 0.2131 & \\
\hline$\Theta$ Breakfast & -0.1395 & \\
\hline
\end{tabular}

The final output of simulation model reveals that the model can replicate the historical trend. The y-axis shows the mental distress variations per day. In the future, we can run the model for a longer period and change the dietary pattern for the policy design purpose (figure 3 ). 


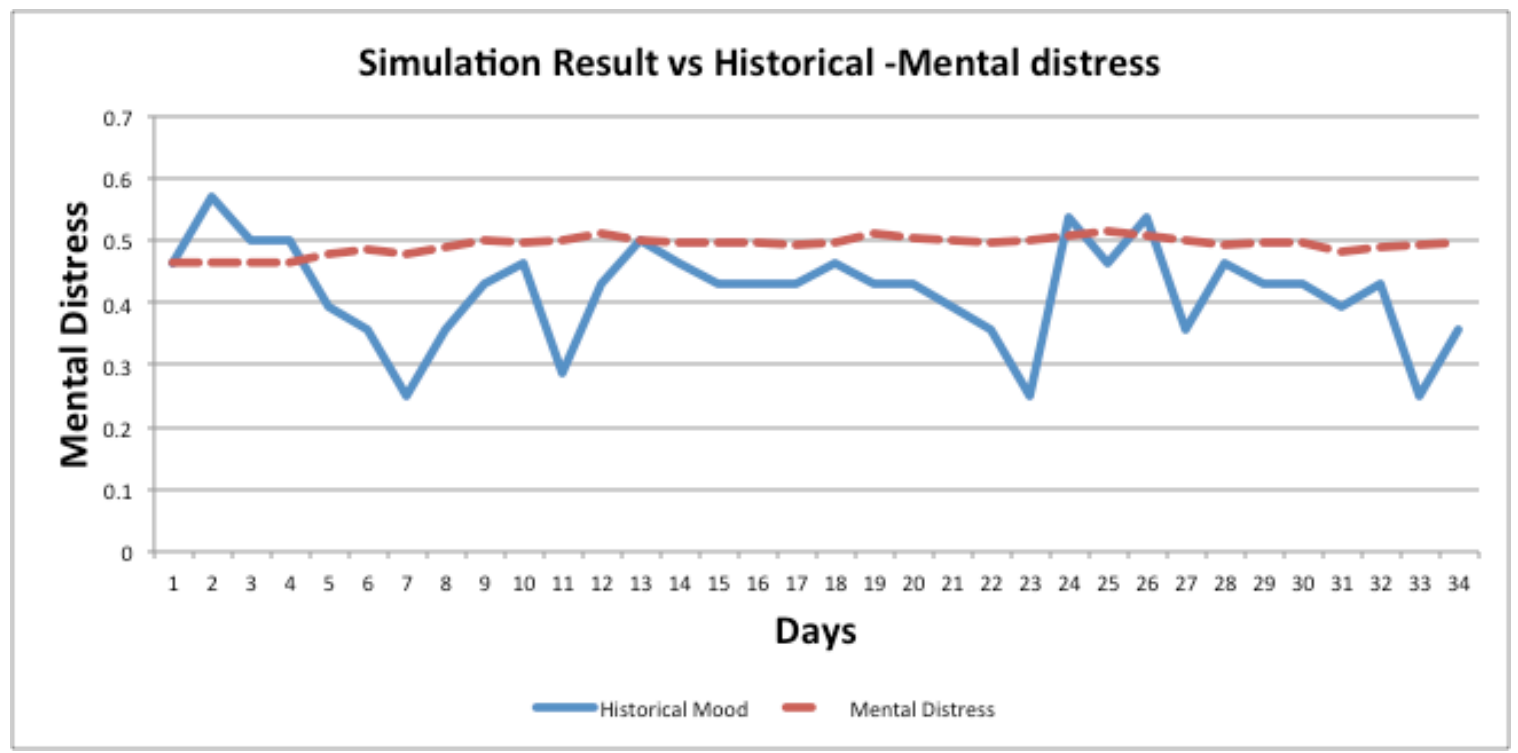

Figure 3 Simulation Result vs. Historical-Mental distress

This the first attempt that we try to map the feedback loop by applying some data mining techniques which are supported by the literature and a previous study [15, 16] . This SD model can provide the environment for policymakers to investigate the impact of different dietary pattern in mental distress and predict the future trend.

\section{Future research}

This study is an ongoing work that we want to find the answer to some questions through applying some machine learning techniques and system dynamics;

1- How can we derive causal relations by using big data analytics and apply it in developing SD models?

2- How can we find nonlinear relationships using longitudinal data for validating SD models?

Future research could identify more casual loops, which relates to depression and anxiety. Using the model we can design new interventions for mental distress prevention. 


\section{References}

1. Seney, M.L. and E. Sibille, Sex differences in mood disorders: perspectives from humans and rodent models. Biology of sex differences, 2014. 5(1): p. 17.

2. Rao, T.S., et al., Understanding nutrition, depression and mental illnesses. Indian Journal of Psychiatry, 2008. 50(2): p. 77.

3. McLean, A., et al., The effects of tyrosine depletion in normal healthy volunteers: implications for unipolar depression. Psychopharmacology, 2004. 171(3): p. 286-297.

4. Rutter, M., A. Caspi, and T.E. Moffitt, Using sex differences in psychopathology to study causal mechanisms: unifying issues and research strategies. Journal of Child Psychology and Psychiatry, 2003. 44(8): p. 1092-1115.

5. Wai, J. and M. Makel, Sex differences in ability tilt in the right tail of cognitive abilities: A 35-year examination. 2017.

6. Ingalhalikar, M., et al., Sex differences in the structural connectome of the human brain. Proceedings of the National Academy of Sciences, 2014. 111(2): p. 823-828.

7. Ruigrok, A.N., et al., A meta-analysis of sex differences in human brain structure. Neuroscience \& Biobehavioral Reviews, 2014. 39: p. 34-50.

8. Tan, A., et al., The human hippocampus is not sexually-dimorphic: metaanalysis of structural MRI volumes. Neuroimage, 2016. 124: p. 350-366.

9. Van Wingen, G.A., et al., Testosterone increases amygdala reactivity in middle-aged women to a young adulthood level. Neuropsychopharmacology, 2009. 34(3): p. 539.

10. Begdache, L., H. Kianmehr, and C.V. Heaney, College Education on Dietary Supplements May Promote Responsible Use In Young Adults. Journal of dietary supplements, 2018: p. 1-14.

11. Hosseinichimeh, N., et al. Estimating system dynamics models using indirect inference. in Proceedings of the International System Dynamics Conference. 2015.

12. Jacka, F.N., et al., Western diet is associated with a smaller hippocampus: a longitudinal investigation. BMC medicine, 2015. 13(1): p. 215.

13. Carrera-Bastos, P., et al., The western diet and lifestyle and diseases of civilization. Research Reports in Clinical Cardiology, 2011. 2: p. 15-35.

14. Erickson, K.I., et al., Exercise training increases size of hippocampus and improves memory. Proceedings of the National Academy of Sciences, 2011. 108(7): p. 3017-3022.

15. Begdache, L., et al., Assessment of dietary factors, dietary practices and exercise on mental distress in young adults versus matured adults: $A$ cross-sectional study. Nutritional neuroscience, 2017: p. 1-11.

16. Begdache, L., et al., Principal component analysis identifies differential gender-specific dietary patterns that may be linked to mental distress in human adults. Nutritional neuroscience, 2018: p. 1-14. 